\title{
Prevalência de Sintomas Osteomusculares em Cuidadores de Pacientes Dependentes
}

\section{Prevalence of Osteomuscular Symptoms in Caregivers of Dependent Patients}

Welyka Mendes Moreira¹, Maria Francisca Lucia dos Santos², Mariane Santos Nogueira $^{3}$, Aleandro Geraldo Alves ${ }^{4}$

\section{RESUMO}

As doenças crônicas vêm aumentando cada vez mais na população levando os indivíduos à dependência, necessitando do auxílio do cuidador. Os cuidadores têm um maior risco de desenvolver distúrbios osteomusculares devido à sobrecarga imposta sobre o cuidado. Os objetivos deste trabalho são analisar a prevalência de sintomas osteomusculares em cuidadores de pacientes dependentes, avaliar presença de formigamento, dor ou dormência no corpo dos cuidadores, avaliar nível de dor e orientar sobre técnicas para prevenção de disfunções osteomusculares. Tratou-se de um estudo descritivo do tipo quantitativo, natureza transversal. A amostra foi composta por 13 cuidadores de pacientes dependentes. Todos os cuidadores foram avaliados através do questionário Nórdico e da Escala Visual Analógica (EVA) e orientados sobre técnicas de mobilização de doentes, segundo Ergonomics for the prevention of musculoskeletal disorders (2003). Houve um número significativo de dor presente nos indivíduos, mas nem todos os cuidadores relatam sentir dores devido ao risco de perderem o emprego, então passam a aceitar a nova realidade. Conclui-se que é de fundamental importância a avaliação de sintomas osteomusculares em cuidadores e um programa de orientações, a fim de evitar agravos à saúde do cuidador de pacientes dependentes.

Palavras-chave: Cuidadores, Dependência Física, Distúrbios Osteomusculares Relacionados ao Trabalho.

\section{ABSTRACT}

Chronic diseases are increasingly increasing in the population, leading individuals to dependence, where the need for caregiver assistance is greatest. Caregivers are at a greater risk of developing musculoskeletal disorders because of the overhead imposed on care. The objectives of this work are analyze the prevalence of musculoskeletal symptoms in caregivers of dependent patients, to assess the presence of tingling, pain or numbness in the caregivers' body, to assess pain level and to advise on techniques for the prevention of western muscle dysfunction. This was a descriptive, quantitative, cross-sectional study. The sample consisted of thirteen caregivers of dependent patients. All the caregivers were evaluated through the Nordic questionnaire and the Visual Analogue Scale and oriented on patient mobilization techniques according to Ergonomics for the prevention of musculoskeletal disorders (2003). There was a significant amount of pain present in the individuals, but not all caregivers report having pain due to the risk of losing their job, then they come to accept the new reality. It is concluded that it is fundamental importance the evaluation of musculoskeletal symptoms in caregivers and a program of guidelines in order to avoid health problems of the caregiver of dependent patients.

Keywords: Caregivers, Physical Dependence, Work-related Musculoskeletal Disorders.
${ }^{1}$ Fisioterapeuta - Faculdade Montes Belos (FMB). Especialista em Docência Universitária FABEC.

E-mail:

welyka_mm15@hotmail.com

${ }^{2}$ Fisioterapeuta - Faculdade Montes Belos (FMB).

E-mail: maria.fls@hotmail.com

${ }^{3}$ Fisioterapeuta - Faculdade Montes Belos (FMB). Especialista em Fisioterapia Neurofuncional CEAFI.

${ }^{4}$ Fisioterapeuta - Faculdades Integradas de Patrocínio. Especialista em Análise e Biomecânica do Movimento Humano - ESEFFEGO.

Especialista em Fisioterapia Traumato-Ortopédica - COFFITO. Mestre em Genética - PUC Goiás. 


\section{INTRODUÇÁO}

Doenças crônicas são definidas como alterações na saúde que se iniciam e desenvolvem gradativamente, tendo como consequência períodos longos de duração e pode manifestar momentos de melhora ou piora (ALMEIDA et al., 2002; MINISTÉRIO DA SAÚDE, 2014).

Atualmente as doenças crônicas são consideradas um grave problema de saúde pública, sendo responsável por $63 \%$ das mortes no mundo. Espera-se que no ano 2020, $80 \%$ das doenças dos países em desenvolvimento devem decorrer de problemas crônicos (OMS, 2011; OMS, 2002).

As causas das condições crônicas vão depender do estilo de vida que o indivíduo apresenta como a sua exposição a fatores ambientais, fisiológicos e genéticos. Geralmente os portadores de doenças crônicas apresentam uma limitação física gerando redução de funcionalidade e de sua qualidade de vida (LORIG et al., 2006; CAMPOLINA, DINI e CICONELLI, 2011; BONETTI, SILVA e TRENTINI, 2013).

Funcionalidade é o bom funcionamento das funções do corpo, onde o indivíduo tem a capacidade e a autonomia de conduzir sua vida independentemente. Já a incapacidade envolve os prejuízos de uma doença como alteração na função corporal, perca da dependência em realizar as atividades de vida diária (AVD's) e dificuldade de interação do indivíduo na sociedade (OMS, 2004). O aumento de doenças crônicas está associado aos avanços da saúde que influenciam na longevidade, progredindo assim para a dependência do indivíduo, o que gera a necessidade do serviço de um cuidador (SARAIVA, 2011).

O cuidador é a pessoa que se disponibiliza para cuidar de indivíduos doentes ou dependentes em suas AVD's com cuidados terapêuticos e preventivos, acompanhamento às consultas, higiene, alimentação, locomoção, saúde e troca de roupas. Esses cuidadores podem ser ou não da família (MINISTÉRIO DA SAUDE, 1999).

O cuidador formal é aquele contratado por uma instituição para prestar assistência por longo prazo, tem seu salário e realiza treinamentos específicos para tais competências e serviços. O informal são pessoas da própria família, vizinhos ou amigos que auxiliam no cuidado e não são remunerados (OMS, 2005).

O trabalho do cuidador vai desde um simples auxílio até a ajuda de profissionais especializados e geralmente são trabalhos que geram uma sobrecarga intensa, e nem sempre têm acesso a informações necessárias para prevenção e tratamento destas 
afecções, tornando-os vulneráveis a desenvolverem problemas de saúde (CARDOSO et al., 2011).

Os problemas de saúde decorrentes do trabalho são responsáveis pela maioria dos casos de afastamento, transferência de função, menor rendimento do serviço, maior custo econômico do empregador, ocorrendo assim queda da qualidade do serviço prestado pelo cuidador (TINUBU et al., 2010).

Os distúrbios osteomusculares constituem condições comuns que afetam os cuidadores. Trata-se de um conjunto de afecções que possuem as mesmas características, com comprometimento de músculos, articulações, ligamentos, nervos, tendões e que surge decorrente ao tipo de atividade realizada no trabalho. Possui um quadro clínico variado como formigamento, dormência, fadiga e dor, esta última se caracteriza como o principal sintoma relatado pelos cuidadores (MINISTERIO DA SAÚDE, 2001; YENG et al., 2001).

Diversos fatores interferem na dor como força excessiva para realizar algumas tarefas, repetitividade de movimentos, longas jornadas de trabalho e posturas inadequadas. As dores na maioria das vezes são relatadas em regiões com maiores sobrecargas como membros superiores, inferiores e coluna. É necessário investigar as cargas em que o trabalhador está exposto, para que sejam realizadas alterações nas condições de trabalho, melhorando a saúde e o bem-estar do cuidador (GUTIÉRREZ, FLORES e MONZÓ, 2010; ALEXOPOULOS, STATHY e CHARIZANI, 2004; FRUTUOSO e CRUZ, 2005).

O nível da sobrecarga está relacionado com o grau de dependência do indivíduo junto às atividades desempenhadas no cuidado. Existem dois tipos de sobrecarga, objetiva e subjetiva. A sobrecarga objetiva é a parte que se nota os problemas do cuidador decorrente ao seu trabalho como estresse, movimentos repetidos e vigilância nos pacientes. A sobrecarga subjetiva é a reação do familiar fazendo o papel do cuidador, onde há mudanças em todos os aspectos de sua vida além da preocupação com o paciente (BANDEIRA, CALZAVARA e CASTRO, 2008; BOCCHI, 2004).

É de extrema importância que os profissionais tenham acesso a informações e orientações sobre os distúrbios osteomusculares, como seus fatores de riscos e complicações, visando assim à prevenção e ao agravamento destas lesões, além de enriquecer o conhecimento destes profissionais para realização de suas atividades laborais. A finalidade destes conhecimentos é obter uma melhora na qualidade de vida do trabalhador para ter um melhor rendimento do serviço prestado por ele (MINISTÉRIO DA SAÚDE, 2008). 
As doenças crônicas aumentam cada vez mais na população e trazem alterações radicais na vida dos indivíduos acometidos, visto que apresentam um tempo de permanência prolongado, necessitando de auxílio de cuidadores. Cuidadores de pacientes dependentes possuem um maior risco de desenvolverem distúrbios osteomusculares, por se tratar de um serviço que exige um maior desgaste físico, e nem sempre têm acesso à informações necessárias para prevenção e tratamento destas afecções. Este estudo justifica-se então, pela prevalência da aplicação de um programa de avaliação de sintomas através do uso de escalas e questionários junto à orientações aos cuidadores a fim de prevenir agravos osteomusculares relacionados ao trabalho, além de enriquecer 0 conhecimento destes profissionais para a realização de suas atividades.

Os objetivos deste estudo foram analisar a prevalência de sintomas osteomusculares relacionados ao trabalho de cuidadores de pacientes dependentes, avaliar presença de formigamento, dor ou dormência no corpo dos cuidadores e, avaliar nível de dor e orientar sobre técnicas para prevenção de disfunções osteomusculares.

\section{MATERIAIS E METODOS}

Foi realizado um estudo descritivo do tipo quantitativo, natureza transversal em cuidadores de pacientes dependentes na instituição Vila São Cotollengo, na cidade de Trindade-GO, onde a amostra foi composta por 13 cuidadores, sendo avaliados através do questionário Nórdico e da Escala Visual Analógica (EVA).

Foram realizadas cinco visitas, sendo quatro para avaliação dos cuidadores e uma para orientações sobre técnicas de mobilização de doentes para prevenção de lesões musculoesqueléticas através de folder informativo. A carta de autorização para coleta de dados foi assinada pela direção da Vila São Cotollengo. As avaliações tiveram cerca de dez minutos cada. O projeto foi apresentado para cada cuidador, onde todas as informações foram esclarecidas e o Termo de Consentimento Livre e Esclarecido (TCLE) foi assinado pelos cuidadores participantes da pesquisa.

Os critérios de inclusão foram cuidadores de indivíduos dependentes, que aceitaram participar da pesquisa e que assinaram o TCLE. E os de exclusão foram cuidadores de indivíduos independentes, que estavam em tratamento médico e/ou fisioterapêutico, que não aceitaram participar da pesquisa e não assinaram o TCLE.

Por se tratar de uma pesquisa onde os indivíduos foram avaliados em seu local de trabalho e receberam orientações, havia a possibilidade de desenvolverem transtornos psicológicos ou qualquer alteração na saúde decorrente à pesquisa realizada. Caso 
apresentassem algum agravo, o cuidador seria encaminhado à atendimento médico e ao psicológico, conforme a carta de autorização para encaminhamento.

O projeto de pesquisa foi encaminhado ao comitê de ética em pesquisa da Faculdade União de Goyazes (FUG), seguiu todas as normas estabelecidas pela Resolução 466/12 do Conselho Nacional de Saúde e teve como protocolo de aprovação o N. 067/2016-2.

Os dados foram analisados por profissional estatístico, por meio do programa estatístico Statistical Package for the Social Sciences (SPSS). Em todas as análises foi adotado o nível de significância estatística de $5 \%(p \leq 0,05)$.

\section{RESULTADOS}

O presente estudo utilizou-se a Escala Visual Analógica (EVA) para análise do nível de dor entre os 13 cuidadores de pacientes dependentes.

O gráfico 1 demonstra que dos 13 cuidadores, 7,7\% relataram dores leves e 46,2\% relataram dores moderada e intensa.

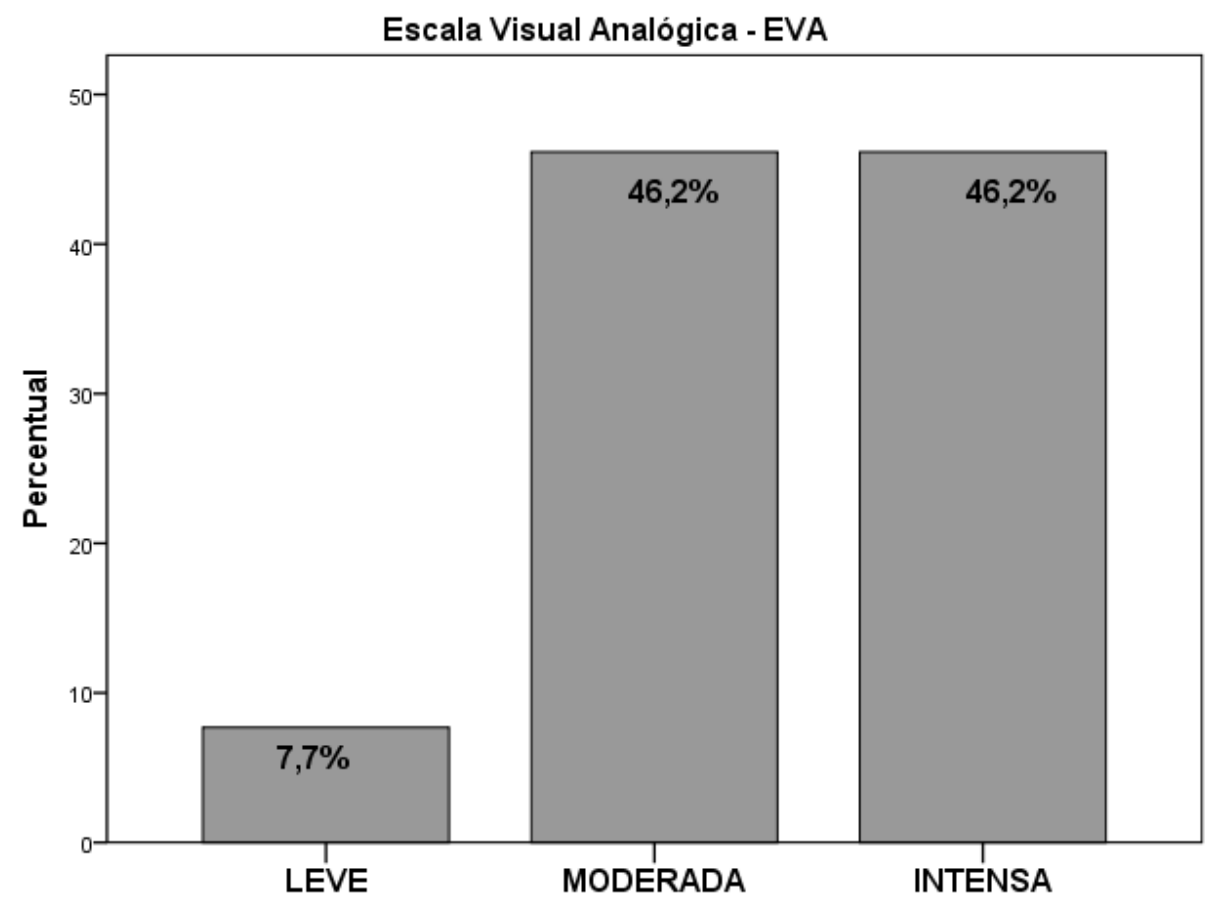

Gráfico 1. Escala Visual Analógica (EVA)

Foi aplicado o questionário Nórdico com a proposta de padronizar a mensuração de relatos de sintomas osteomusculares em diversas regiões anatômicas dos cuidadores.

Conforme mostra o gráfico 2, os cuidadores relataram presença de dor, formigamento/dormência nos últimos 12 meses em diversas áreas do corpo. 46,2\% dos 
cuidadores relataram não ter sintomas na região do pescoço, parte superior das costas, punho/mãos e 53,8\% relataram presença dos sintomas nestas regiões anatômicas. 38,5\% relataram não ter sintomas em ombros e parte inferior das costas e 61,5\% relatam a presença dos sintomas nestas regiões anatômicas. 61,5\% relataram não ter sintomas em cotovelos e tornozelos/pés e 38,5\% relataram a presença dos sintomas. 53,8\% relataram não ter sintomas em quadril/coxas e $46,2 \%$ relataram a presença dos sintomas nestas regiões anatômicas. 76,9\% relataram não ter sintomas em joelhos e 23,1\% relataram a presença dos sintomas nestas regiões.

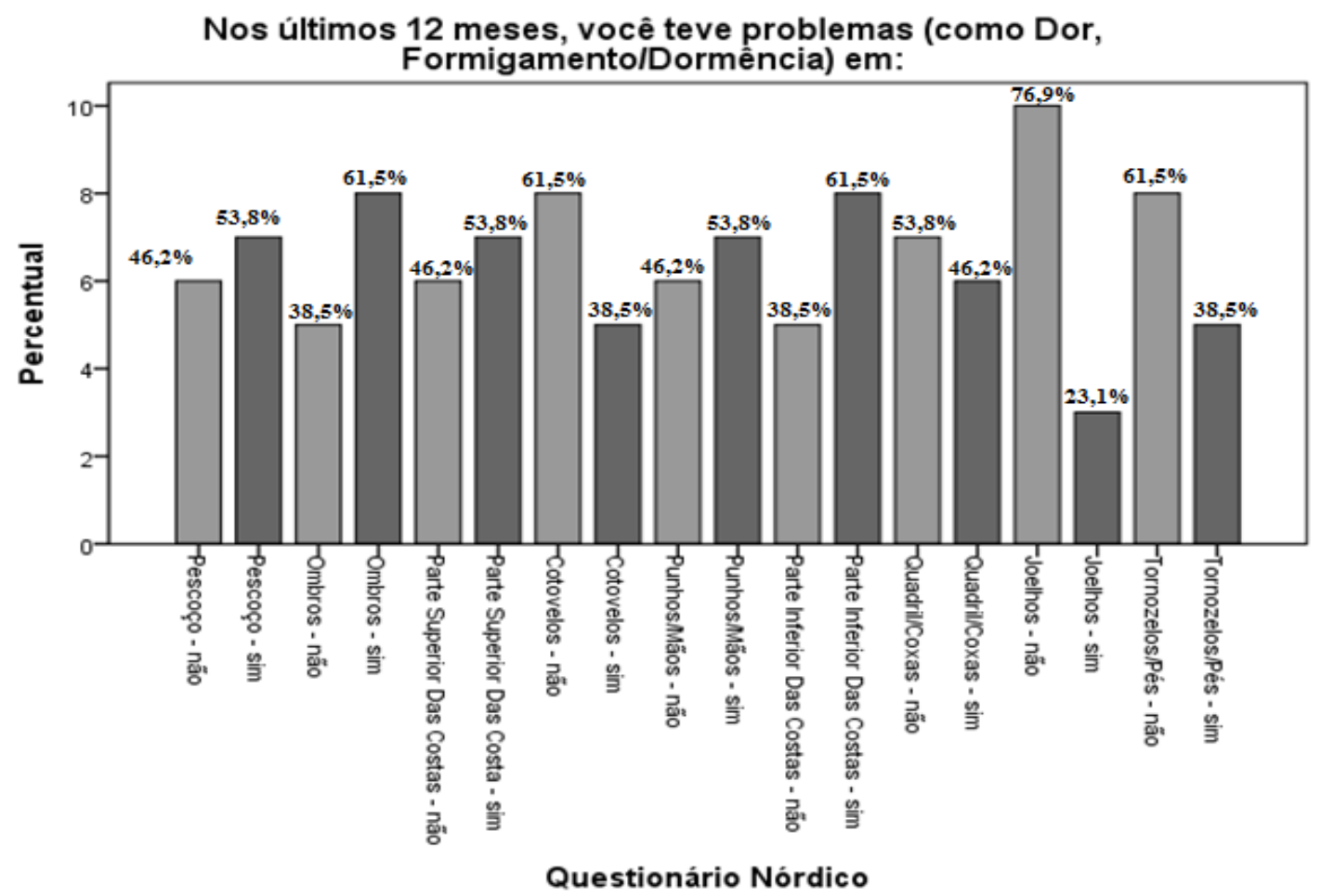

Gráfico 2. Sintomas nos últimos 12 meses

Os cuidadores responderam se nos últimos 12 meses foi impedido de realizar atividades normais como trabalho, atividade doméstica e de lazer por causa dos sintomas nas regiões anatômicas avaliadas.

O gráfico 3 nos mostra que 92,3\% dos cuidadores não foram impedidos de realizar suas atividades devido aos sintomas na região do pescoço, parte superior das costas, punho/mãos, joelhos e tornozelos/pés e em $7,7 \%$, os sintomas impediram de realizar suas atividades nestas regiões. $84,6 \%$ não foram impedidos de realizar suas atividades devido aos sintomas na região dos ombros, cotovelos e quadril/coxas e 15,4\%, os sintomas nestas 
regiões impediram de realizar as atividades. 76,9\% não foram impedidos de realizar suas atividades devido aos sintomas na parte inferior das costas e em 23,1\% os sintomas impediram de realizar suas atividades.

Nos últimos 12 meses, você foi impedido(a) de realizar atividades normais (por exemplo: trabalho, atividades domesticas e de lazer) por causa desse problema em:

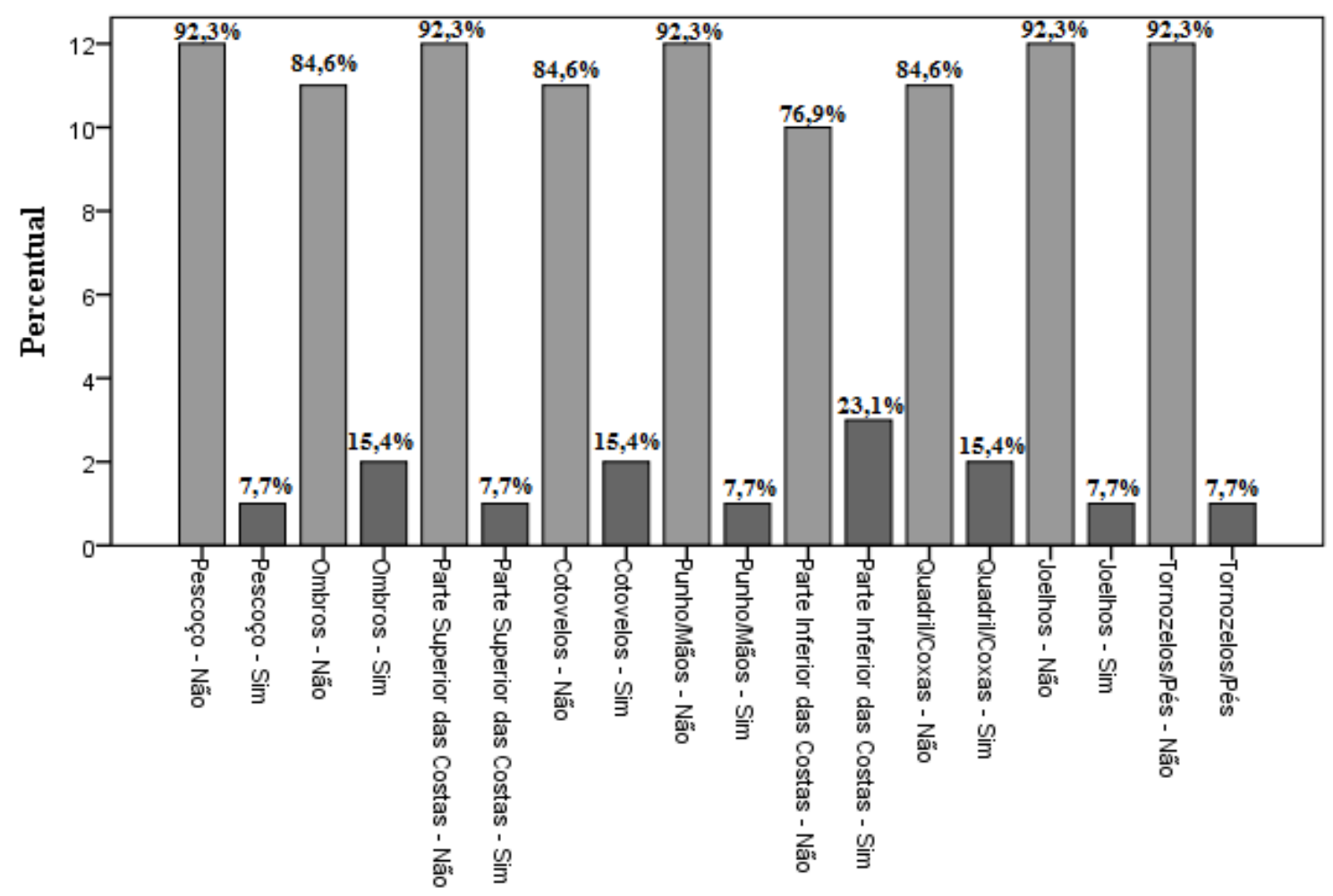

Questionário Nórdico

Gráfico 3. Impedimento de realizar as atividades do dia a dia

Foi questionado aos cuidadores se nos últimos 12 meses, eles consultaram algum profissional da saúde como médico, fisioterapeuta por causa dos sintomas já referidos.

Observa-se no gráfico 4 que 76,9\% dos cuidadores não procuraram ajuda de profissionais da saúde devido aos sintomas na região do pescoço, ombros, parte superior das costas, cotovelo, parte inferior das costas e quadril/coxas e 23,1\% consultaram algum profissional na área da saúde devido aos sintomas nas regiões citadas. 84,6\% não procuraram ajuda de profissionais da saúde devido aos sintomas na região do punho/mãos, joelhos e tornozelos/pés e 15,4\% consultaram algum profissional na área da saúde devido aos sintomas citados. 
Nos últimos 12 meses, você consultou algum profissional da área da saúde (médico, fisioterapeuta) por causa dessa condição em:

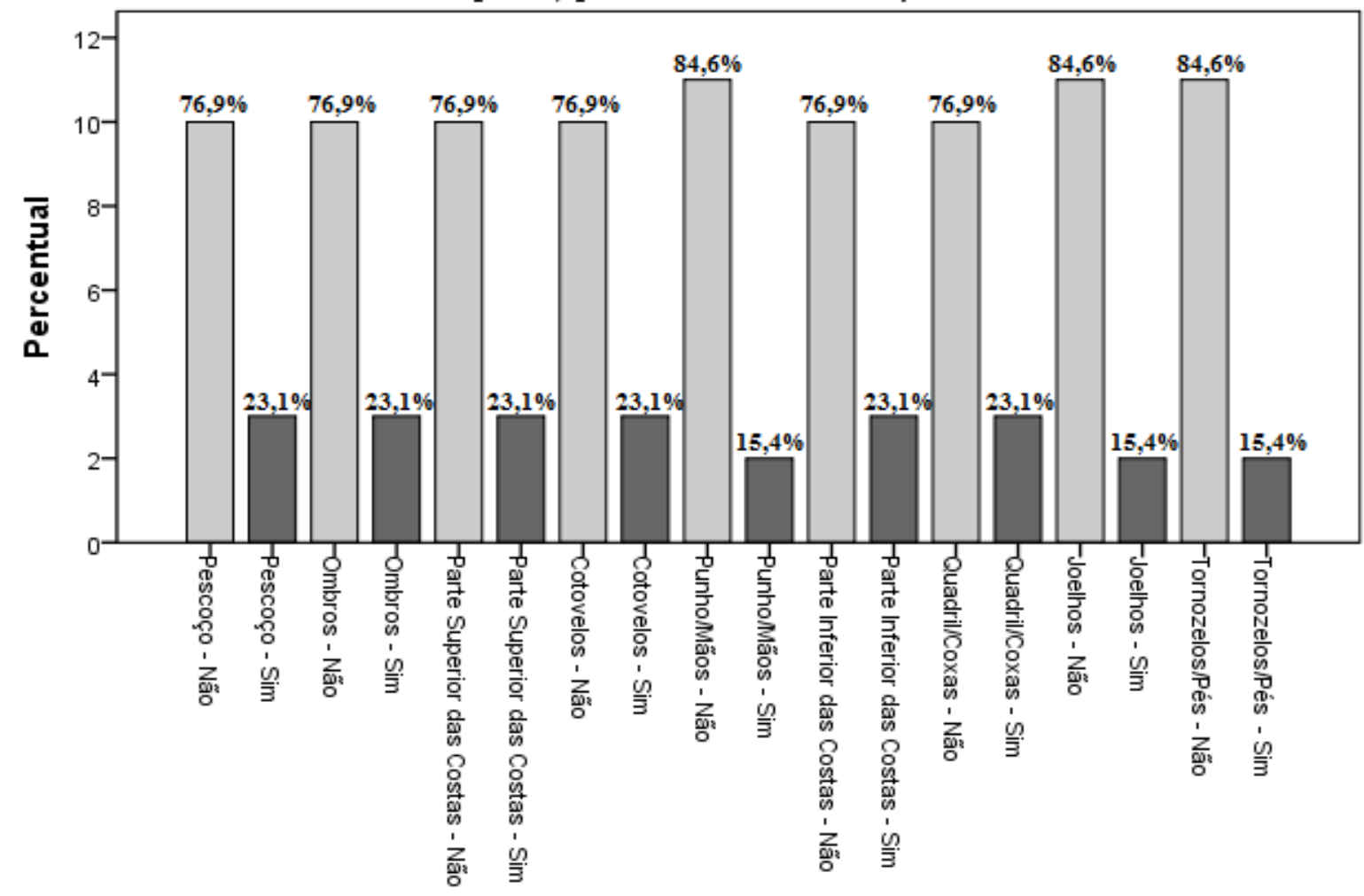

Questionário Nórdico

Gráfico 4. Procura de algum profissional da saúde

Os cuidadores relataram se nos últimos sete dias, tiveram algum problema nas partes anatômicas avaliadas.

O resultado do gráfico 5 demonstrou que $92,3 \%$ dos cuidadores relataram que não tiveram algum problema na região do pescoço, cotovelo, quadril/coxas, joelhos e tornozelos/pés e 7,7\% dos cuidadores relataram ter tido problemas nestas partes do corpo nos últimos sete dias. $84,6 \%$ dos cuidadores relataram que não tiveram problema na região dos ombros, punho/mãos e 15,4\% relataram ter tido problemas nestas partes do corpo nos últimos sete dias. $69,2 \%$ dos cuidadores relataram que não tiveram problema na parte superior das costas e 30,8\% relataram ter tido problemas nessa parte do corpo nos últimos sete dias. $23,1 \%$ dos cuidadores relataram que não tiveram problema a parte inferior das costas e $76,9 \%$ relataram ter tido problemas nessa parte do corpo nos últimos sete dias. 


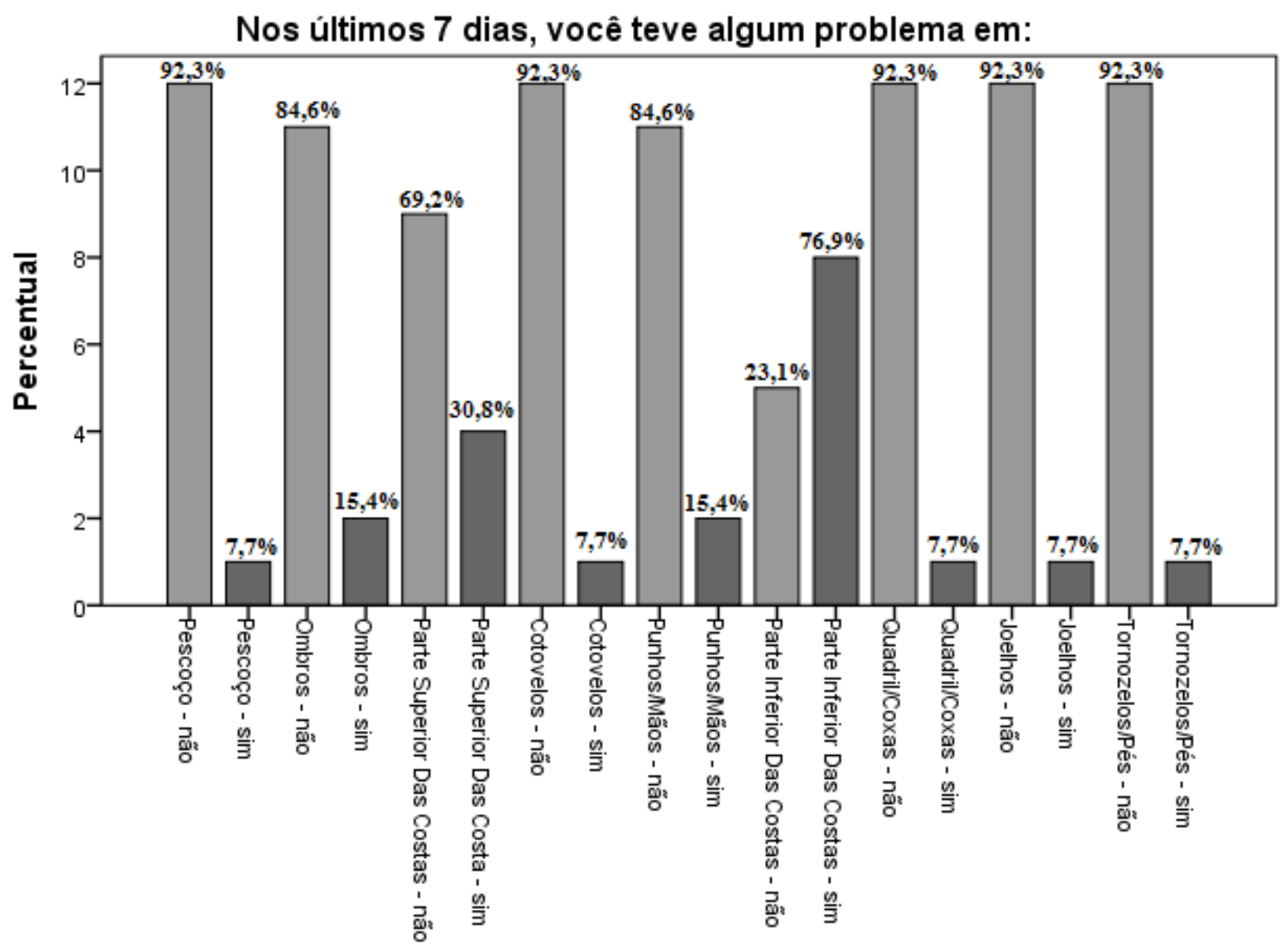

Questionário Nórdico

Gráfico 5. Algum problema nos últimos sete dias.

\section{DISCUSSÃO}

Neste estudo foi realizada uma avaliação de presença e nível de sintomas osteomusculares em indivíduos através da Escala Visual Analógica e do Questionário Nórdico. Estes instrumentos são importantes, pois fornecem informações para melhor avaliar a sintomatologia presente nos indivíduos. Barros (2004); Borges (2003) e Simpionato (2005), afirmam que conhecer quais as situações de risco em que os cuidadores estão expostos pode ajudar a prevenir as alterações à saúde e evitar que afete a qualidade de vida tanto do cuidador quanto do paciente.

Em relação às características da amostra, os cuidadores apresentaram nível significativo de dor. Os sintomas dolorosos agravam-se progressivamente e podem evoluir para perda da funcionalidade. De acordo com Costa et al., (2013) a presença de dor está associada ao desconhecimento para realizar o cuidado, fatores biomecânicos e posturais, níveis de demanda requeridos pelo dependente, àqueles que prestam assistência nas tarefas de banho, vestimenta, alimentação e transferências estão expostos a maior 
sobrecarga e tensão. Quanto mais comprometida sua autonomia, maiores são as demandas e a complexidade das atividades desenvolvidas pelo cuidador.

Foi verificada na presente pesquisa um índice maior de dores na coluna cervical. Para Coury, Moreira e Dias (2009) os sintomas osteomusculares na coluna cervical têm relação com sua alta flexibilidade, capacidade de executar variados movimentos e com posturas inadequadas.

Perante este estudo nota-se alta prevalência de dor nos ombros. Junior e Assunção (2005) relatam que os distúrbios do ombro estão associados à manutenção de posturas estáticas por tempo prolongado ao prestar assistência, movimentos repetitivos de braço, elevação e abdução dos braços acima dos ombros e ainda cargas excessivas em membros superiores.

Como observado nos resultados da pesquisa atual, houve um considerável número de dor na região dorsal superior. Gurgueira, Alexandre e Filho (2003) realizaram um estudo utilizando o Questionário Nórdico, avaliaram auxiliares e técnicos de enfermagem que trabalhavam em enfermarias que havia pacientes dependentes. Os participantes da pesquisa relataram sentir dor na região dorsal superior principalmente no momento de transferir o paciente para a maca/cadeira de rodas e vice-versa, dar banho no leito e que não possuíam técnicas atuais de transferência para realizar o devido cuidado, o que pode explicar a alta prevalência de dor dorsal entre os estudos.

Grande parte dos participantes da pesquisa relatou presença de dor na coluna lombar. Segundo Barbosa e Gonçalves (2007) e Maia et al., (2008) a lombalgia é uma das doenças mais frequentes no mundo industrializado e gera impactos não apenas na saúde física, mas também interfere na funcionalidade e qualidade de vida dos indivíduos. A coluna lombar tem um maior índice de acometimento sintomatológico pelo fato da movimentação de pacientes e manutenção da posição em flexão, fadigas musculares e sobrecargas excessivas sobre estruturas da coluna vertebral lombar como discos intervertebrais e ligamentos.

Apesar do presente estudo mostrar níveis consideráveis de dor entre os cuidadores, os autores Yamashita et al., (2010) relatam que alguns cuidadores negam qualquer sintomatologia decorrente ao seu trabalho, pelo fato de não haver perspectiva de mudança de função e precisarem do emprego, ocorre então um ajustamento frente à continuidade da dor, sugerindo um comportamento de aceitação ou adaptação à nova realidade.

Os resultados do estudo atual mostram que as chances do cuidador ser impedido de realizar suas atividades do dia a dia devido aos sintomas osteomusculares provocados pelo 
trabalho são mínimas. Mas em seu estudo, Alexandre (2007) afirma que cuidadores de pacientes dependentes estão suscetíveis a alterações funcionais pelo fato da sobrecarga física e também do estress emocional, influenciando assim na sua qualidade de vida.

Foram realizadas neste estudo, orientações quanto a técnicas de mobilização de doentes para a prevenção de lesões musculoesqueléticas. Nota-se que o sucesso de uma transferência depende da postura do cuidador, sendo ela importante no momento de prevenção de distúrbios osteomusculares. Bazo e Gimenezes (2008) e Fernandes et al., (2013) afirmam que programas de prevenção de distúrbios osteomusculares devem ser criados a fim de evitar que a saúde do cuidador seja atingida negativamente proveniente ao trabalho realizado por ele. A prevenção de distúrbios osteomusculares e a manutenção de uma boa qualidade de vida nos cuidadores são importantes, quanto mais sadios eles estiverem mais aptos estarão para executar suas atividades e atender às necessidades de seus doentes.

\section{CONSIDERAÇOES FINAIS}

Com este estudo observou-se que o cuidador tem um papel fundamental na vida do paciente dependente, mas, suas atividades podem sobrecarregá-lo pelo despreparo em relação ao papel que desempenha, e isso poderá causar alterações em sua saúde até a perca da funcionalidade. Sendo assim, é necessário avaliar o trabalho do cuidador e a sobrecarga imposta pelo mesmo.

Além disso, analisar a presença de dor, formigamento, dormência e a intensidade destes sintomas osteomusculares em cuidadores é essencial para diminuir a possibilidade de agravos a saúde, visto que os cuidadores da pesquisa atual possuíam uma prevalência significativa de sintomas osteomusculares.

Verifica-se então a importância de um programa de prevenção de distúrbios osteomusculares por meio de orientações aos cuidadores de pacientes dependentes.

Dessa forma, diante do que foi exposto, esta pesquisa enfatiza que o cuidador necessita de cuidados, para que uma assistência em saúde, humanizada e integral, seja alcançada. 
ALMEIDA, Marcia Furquim; BARATA, Rita Barradas; MONTERO, Cláudia Valencia; SILVA Zilda Pereira. Prevalência de doenças crônicas autoreferidas e utilização de serviços de saúde, PNAD/1998, Brasil. Ciência \& Saúde Coletiva, São Paulo, 2002, p. 743 - 746.

BANDEIRA, Marina; CALZAVARA, Maria Glaucia Pires; CASTRO, lidevane. Estudo de validade da escala de sobrecarga de familiares cuidadores de pacientes psiquiátricos. J. Bras. Psiquiatr. Tiradentes, 2008, p. 98 - 104.

BARBOSA, Fernando Sergio Silva; GONÇALVES, Mauro. A proposta biomecânica para a avaliação de sobrecargas na coluna lombar: efeito de diferentes variáveis demográficas na fadiga muscular. Acta Ortop Bras, v. 3, 2007, p. 132-137.

BARROS, Tania Martins. Doença renal crônica: do doente e da dimensão familiar. São Paulo: Casa do psicólogo, 2004.

BAZO, Márcia Lali; GIMENEZ, Brigida Carvalho. Caracterização das alterações dos cuidadores do PSF da unidade de saúde do Ouro Branco/Londrina. Ciênc. Biol. Saúde, v. 10, n. 1,2008, p. 51-58.

BOCCHI, Silva Cristina Mangini. Vivenciando a sobrecarga ao vir-a-ser um cuidador familiar de pessoa com acidente vascular cerebral (avc): uma análise do conhecimento. Rev. Latino - Am Enfermagem, São Paulo, v. 2, 2004, p. 115 - 21.

BONETTI, Albertina; SILVA, Denise Guerreiro V; TRENTINI, Mercedes. O método da pesquisa convergente assistencial em um estudo com pessoas com doença arterial coronariana. Esc Anna Nery, Rio de Janeiro, v.17, 2013, p.179 - 183.

BORGES, Cláudia Fernandes. Dependência e morte da "mãe de família": a solidariedade familiar e comunitária nos cuidados com a paciente de esclerose lateral amiotrófica. Psicologia em Estudo, Maringá, v. 8, 2003, p. 21- 29.

BRASIL, MINISTÉRIO DA SAÚDE. Lesões musculoesqueléticas relacionadas com trabalho: Guia de orientação para prevenção. Portugal, 2008.

BRASIL, MINISTÉRIO DA SAÚDE. Lesões por esforços repetitivos (Ler) e Distúrbios osteomusculares relacionados ao trabalho (DORT). Brasília: Ministério da Saúde, 2001.

BRASIL, MINISTÉRIO DA SAÚDE. Política nacional do idoso. Portaria n.1395/GM. Brasília, 1999.

BRASIL, MINISTÉRIO DA SAÚDE. Portal da Saúde. Brasília, 2014.

CAMPOLINA, Alessandro Gonçalves; DINI, Patrícia Skolaude; CICONELLI, Rosana Mesquita. Impacto da doença crônica na qualidade de vida de idosos da comunidade em São Paulo (SP, Brasil). Revista Ciência e Saúde Coletiva, São Paulo, v. 16, n. 6, 2011.

CARDOSO, Lucilene; VIEIRA, Verderoce Mariana; RICCI, Maira Aparecida Malagutti; MAZZA, Rafael Siverio. Perspectivas atuais sobre a sobrecarga do cuidador em saúde mental. Rev Esc Enferm USP, São Paulo, v. 46, n. 2, 2012. 
COSTA, Erica Cristiane Sousa; PEREIRA, Poliana Delfino; MIRANDA, Rodrigo Aparecido Prates; BASTOS, Victor Hugo Vale; MACHADO, Diones Dutra Castro. Sobrecarga física e mental dos cuidadores de pacientes em atendimento fisioterapêutico domiciliar das estratégias de saúde da família de diamantina (MG). Revista Baiana de Saúde Pública Diamantina, v. 37, n. 1, 2013.

COURY, Helenice Januario; MOREIRA, Roberta Farias; DIAS, Natália Bastos. Efetividade do exercício físico em ambiente ocupacional para controle da dor cervical, lombar e do ombro: uma revisão sistemática. Revista Brasileira de Fisioterapia, São Carlos, v. 13, n. 6,2009 , p. 461-79.

FERNANDES, Bruna Cristina; FERREIRA, Karla Pacheco; MORODIN, Maira Franciele; VAL, Maria Odette; FREZ, Andersom Ricardo. Influência das orientações fisioterapêuticas na qualidade de vida e na sobrecarga de cuidadores. Fisioter. Mov, v. 26, n.1, 2013, p. 151-158.

FRUTUOSO, Joselma Tavares; CRUZ Roberto Morais. Mensuração da carga e sua relação com a saúde do trabalhador. Revista Brasileira Medicina do Trabalho, Belo Horizonte, v. 3, n. 1, 2005, p. 29-36.

GURGUEIRA, George Pires; ALEXANDRE, Norton Morais; FILHO, Henrique, Corrêa. Prevalência de sintomas músculo-esqueléticos em trabalhadores de enfermagem. Rev. Latino-Am Enfermagem, v. 11, n. 5, 2003.

GUTIÉRREZ, Manuel Henriquez; FLORES, Carol Rivera; MONZÓ Jorge Eyzaguirre. Prevalencia de Transtornos Músculo-Esqueléticos de Columna Lumbaren Trabajadoras y Límites Biomecánicos enel Manejo de Carga y Pacientes. Estrucplam, Concepción, 2010.

JUNIOR, Hélio Pires Mendonça; ASSUNÇÂO, Ávila Ada. Associação entre distúrbios do ombro e trabalho: breve revisão da literatura. Rev. Bras Epidemiol, v. 8, n. 2, 2005.

LORIG, Kate; HOLMAN, Halsted; SOBEL, David; LAURENT, Diana; GONZALEZ, Virginia; MINOR, Marian. Living a Healthy Life with Chronic Conditions: Self Management of Heart Disease, Arthritis, Diabetes, Asthma, Bronchitis, Emphysema and others. $3 \mathrm{Ed}$. Boulder: Bull Publishing Company, 2006.

MAIA, Angélica Campos; FIALHO, Bruno Camila; ALCÂNTARA, Marcus Alessandro; MORAIS, Rosane Luzia Souza. Incapacidade funcional associada à lombalgia em cuidadores de crianças com paralisia cerebral grave. Fisioterapia e Pesquisa, São Paulo, v. 15, n. 14, 2008, p. 349-54.

ORGANIZAÇÃO MUNDIAL DE SAÚDE. Classificação Internacional de funcionalidade, incapacidade e saúde. Direção geral da saúde. Lisboa, 2004.

ORGANIZAÇÃO MUNDIAL DE SAÚDE. Cuidados inovadores para condições crônicas. Brasília, 2002.

ORGANIZAÇÃO MUNDIAL DE SAÚDE. Envelhecimento ativo: uma política de saúde. Brasília, 2005. 
ORGANIZAÇÃO MUNDIAL DE SAÚDE. Plano de ações estratégicas para o enfrentamento das doenças crônicas não transmissíveis (DCNT) no Brasil 2011 2022. Brasília, 2011.

SARAIVA, Dulce Maria Ferraz. O olhar dos e pelos cuidadores: Os impactos de cuidar e a importância do apoio ao cuidador. Coimbra, vol. 1, 2011. Tese (Mestrado em intervenção social, inovação e empreendedorismo, apresentada à Faculdade de Psicologia e Ciências da educação e à Faculdade de Economia).

SIMPIONATO, Erica. A enfermagem familiar na promoção da saúde de famílias de crianças com insuficiência renal crônica. Ribeirão Preto, 2005. (Dissertação apresentada à Escola de Enfermagem de Ribeirão Preto da Universidade de São Paulo, para obtenção de título de Mestre em enfermagem em Saúde Pública).

TINUBU, Bolanle MS; MBADA, Chidozie; OYEYEMI, Adewale; AFABUNMI, Ayodele. WorkRelated Musculoskeletal Disorders among Nurses in lbadan, South-west Nigeria: a crosssectional survey. BMC Musculoskeletal Disorders, Nigeria, 2010.

YAMASHITA, Cintia Hitomi; AMENDOLA, Fernanda. Perfil sociodemográfico de cuidadores familiares de pacientes dependentes atendidos por uma unidade de saúde da família no município de São Paulo. O Mundo da Saúde, São Paulo, v. 1, 2010, p. 20-24.

YENG, Lin Tchia; TEIXEIRA, Manoel Jacobsen; ROMANO, Miriam; PICARELLI, Helder; SETTIMI, Maria Maeno; GREVE, Julia Andrea. Distúrbios ósteo-musculares relacionados ao trabalho Cummulative trauma disorders. Rev. Med, São Paulo, v. 80, 2001. 